\title{
Experiences and satisfaction of high-risk mothers who gave birth in select facilities in Legazpi City, Philippines
}

\author{
Maria Stephanie Fay S. Cagayan", Veincent Christian F. Pepito ${ }^{2}$, Rita Mae Ang-Bon ${ }^{3}$, \\ Erlidia F. Llamas-Clark ${ }^{4}$ \\ ${ }^{1,2,4}$ College of Medicine, University of the Philippines Manila, Philippines \\ ${ }^{2}$ School of Medicine and Public Health, Ateneo de Manila University, Philippines \\ ${ }^{3,4}$ Regional Office V, Department of Health, Philippines
}

\section{Article Info \\ Article history: \\ Received Nov 8, 2019 \\ Revised Mar 10, 2020 \\ Accepted Apr 24, 2020}

\section{Keywords:}

Childbirth

Patient satisfaction

Pregnancy

Quality of care

Service delivery networks

\begin{abstract}
Among the interventions implemented to curb maternal mortality in the Philippines, service delivery networks (SDNs) have been one of the most important. However, due to their recent implementation, frameworks to assess the quality of care they provide have not yet been established. To address this need, we had formulated the Integrated Patient-Centered Health Service Framework and used it to explore the satisfaction of mothers who gave birth in select facilities Legazpi City Philippines. We conducted key-informant interviews with 14 mothers. We found out that they were satisfied with the quality of care during pre-pregnancy, pregnancy, and post-partum; however, the quality of care during labor and delivery, adherence to referral protocols, and respect for patient's autonomy can still be improved. The framework should be used by program managers to have a qualitative measure of quality of care provided by SDNs, as well as to ensure that referral protocols were implemented.
\end{abstract}

This is an open access article under the CC BY-SA license.

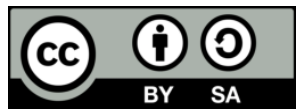

\section{Corresponding Author:}

Maria Stephanie Fay S. Cagayan,

College of Medicine,

University of the Philippines Manila,

Pedro Gil St., Ermita, 1000 Manila, Philippines.

Email: mscagayan@up.edu.ph

\section{INTRODUCTION}

Several low and middle-income countries have failed to meet their millennium development goals (MDG) targets for the reduction of maternal mortality [1], including the Philippines. As of 2015, the country's maternal mortality ratio (MMR) still stands at 114 per 100,000 live births, which demonstrated a slow reduction from the 1990 baseline of 152 per 100,000 live births [2]. This is despite the multitude of public health programs and policies that were implemented from the national to the local level to address this problem [3-7]. However, a cursory look at the implementation dates of these policies reveal that such interventions were not implemented sooner for it to contribute significantly in decreasing maternal mortality.

Poor quality of and the inadequacy of the continuity of care across the health system are among the key contributors to the burden of maternal mortality in the Philippines $[8,9]$. To address this problem, service delivery networks (SDNs), defined as "a network of health facilities and providers within province or city-wide health system, offering core packages of reproductive healthcare services in an integrated and coordinated manner" were implemented across the country. SDNs contain formal agreements between different stakeholders in a local health system that would detail their roles to be played for every possible situation that could arise during family planning, pregnancy, childbirth, and postpartum. These agreements 
include, but are not limited to: Empowering local government units (LGUs) to establish their own city-wide or province-wide SDNs by inviting and overseeing a comprehensive referral system involving primary care, secondary care and tertiary care facilities, as well as both private and public (government) facilities, and; Establishing a local community-based transport and communication system [10].

SDNs were only rolled-out on 2014 [10], with varying extents of implementation. As a result, there is paucity of literature assessing how this intervention influence maternal outcomes and the quality of care that they provide. This, in turn, may stem from the absence of a framework that could be used to assess the provision of care by these SDNs. Notwithstanding, this emphasis on quality of care, especially in recent years, is due to an ongoing paradigm shift in the measurement of pregnancy outcomes from a purely biomedical approach to a more inclusive one that considers the experiences of mothers themselves [11]. This entails the need for a holistic framework that also considers the quality of care being received while the mother progresses from pre-pregnancy, to pregnancy, to labor and delivery, and to postpartum. To this end, the Person-Centered Care Framework for Reproductive Health Equity [12] has been proposed. However, this framework did not emphasize enough the roles being played by SDNs, and the interconnectedness of primary, secondary, and tertiary care throughout the continuum of care for pregnant women. Furthermore, they considered the attainment of person-centered outcomes as an end in itself, rather than as a means to attain the Sustainable Development Goals by improving quality of care. Thus, building on their framework, together with other established frameworks such as the eight principles of patient-centered care proposed by the Picker Institute [13], we are proposing the integrated patient-centered health service framework for maternal health shown in Figure 1.

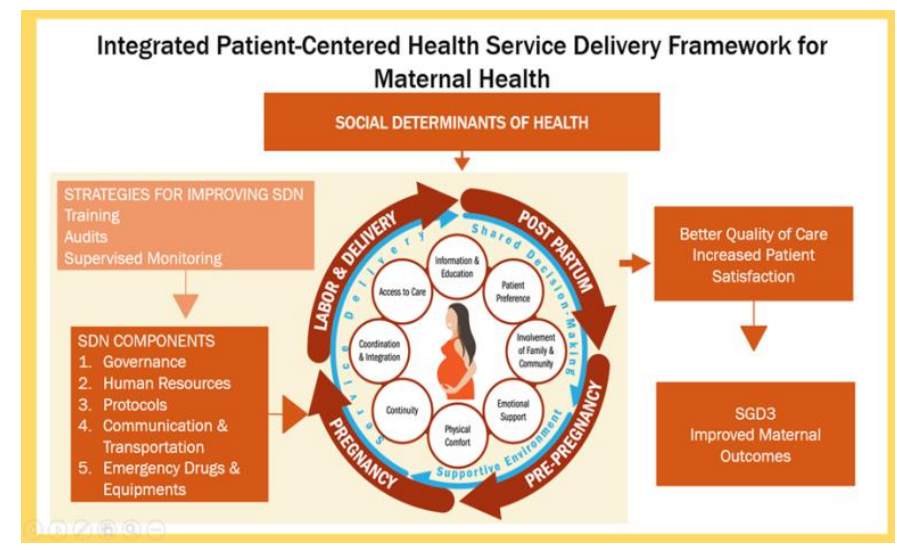

Figure 1. Integrated patient-centered health service framework for maternal health

The integrated patient-centered health service framework for maternal health shows how the different principles of patient-centered care interact with one another throughout the continuum of care for pregnancy. The eight main principles of patient-centered care can be combined into three overlapping themes: shared decision-making, which emphasizes the need for both healthcare provider and patient to play an active role in making decisions regarding the health of the latter; supportive environment, which highlights the role being played by caregivers, family, and community; and service delivery, which underscores access and continuity of quality care from primary to tertiary care, and throughout the continuum of care for pregnancy. This framework also shows the interaction of these principles as an output as a result of inputs to improve the various SDN components. Most importantly, this framework emphasizes that a patient-centered perspective is a means to improve the quality of care and patient satisfaction of pregnant mothers, which would translate to decreasing maternal morbidity and mortality. The social determinants of health are seen to affect every aspect of the continuum of care.

Using this framework as the theoretical lens, this study aims to document and explore the satisfaction of high-risk mothers who gave birth in select facilities in Legazpi City, Philippines. Specifically, this study aims to describe the experiences and satisfaction of the mothers with the quality of care provided by the SDNs in terms of the three main themes of patient-centered care according to the Framework: shared decision making, supportive environment, and service delivery. By doing so, a baseline, qualitative assessment of the quality of care provided by these SDNs will be obtained. This can be used for setting standards for quality of care in these SDNs, especially in other low and middle income countries, which would translate to better maternal outcomes in these settings. 


\section{RESEARCH METHOD}

The study utilized a qualitative phenomenological design to explore the experiences and assess the satisfaction of mothers to the quality of care provided to them with the integrated patient-centered health service framework for maternal health as the theoretical framework.

\subsection{Study design}

The study population is purposively-sampled high-risk mothers who gave birth at select facilities in Legazpi City, Philippines from January to June 2018 who consented to participate in this study. For this study, 'high-risk' is defined as having had the following medical conditions and/or complications during their last pregnancy that will indicate these mothers for referral to a facility with specialist or subspecialist services: hypertension and diabetes (both chronic and gestational); obstetric hemorrhage; sepsis; endometritis; heart disease; diabetes mellitus; delivering pre-term; having respiratory distress; infections (e.g. teratogenic infections, sexually-transmitted infections, etc.); eclampsia and pre-eclampsia, and mothers giving birth for the first time.

\subsection{Study site}

The study was carried out in Legazpi City, Philippines. It is the capital city of the province of Albay, and is the largest city in Bicol Region. The city has a population of 196,639 as of the 2015 Philippine Census [14]. For the same year, the region has registered a maternal mortality ratio (MMR) of 123.5 per 100,000 live births; however, the MMR for Legazpi City during the same time period is only 6.1 per 100,000 live births [15]. Despite its low MMR, Legazpi City was chosen as the study site due to the presence of operational SDNs in the city, and because the largest secondary and tertiary hospitals in the region are located there. The city also has four barangay health centers with birthing stations, 12 private lying in-clinics, and five secondary and/or tertiary hospitals. This would give a clear picture of the health system in a region with high MMR. Furthermore, it will also show the satisfaction of patients with the obstetric services they receive as they move from primary to secondary or tertiary care.

The interviews were conducted predominantly on the health centers or lying-in clinics where these mothers usually consult, although interviews were carried out in the residences of the mothers when they cannot come to the health center. When interviews are being conducted in the health center, the attending midwife or her colleagues, or their other patients, may have been present. If conducted at home, the children, as well as the partners of the respondent mothers, may have been present.

\subsection{Data collection}

Using a semi structured interview guide, we performed key-informant interviews in Filipino, the national language, with the women who gave informed consent to participate in the study. Socio-demographic and other pertinent characteristics such as age, address, gravidity and parity, educational attainment, healthcare provider who initially attended to them during delivery, time from home to center, indication for referral, mode of transportation from their home to the center, destination facility, outcome of pregnancy, and whether or not they were given a discharge summary (i.e., tagubilin) at the time of their last pregnancy were obtained. Participants were also asked to describe their experiences during pre-natal check-ups, labor, and postpartum during their previous pregnancies. The highlight of the interview was the narration of their satisfaction with the care and services they received every step of the continuum of care from pregnancy to post-partum for the latest pregnancy. The proceedings of these key-informant interviews were audio-recorded.

\subsection{Data analysis}

The audio recordings were transcribed and anonymized. After this, the transcriptions were translated to English. The translated transcriptions were then analyzed thematically to identify parts of the mothers' narratives that describe their experiences and satisfaction of the quality of care provided to them according to the following main themes: shared decision-making, supportive environment, and service delivery. Parts of their narratives which would describe instances where their opinion as a patient was considered in their health choices, or lack thereof, would be categorized under Shared Decision-Making. Segments of their stories which would describe involvement and support by their spouses, families, and caretakers towards provision of care would be coded under Supportive Environment. Descriptions of the access, continuity, and quality of medical care to include availability of infrastructure, skilled health personnel, and protocols as well as respectful maternity care were coded under Service Delivery. We independently coded all the transcripts, and any disputes were resolved through discussion. Coding was carried out in NVivo 12 [16].

\subsection{Ethics}

This study received ethical clearance from the University of the Philippines Manila Research Ethics Board (UPM REB 2018-010-01). 


\section{RESULTS AND ANALYSIS}

Fourteen high-risk mothers were invited to participate in the study, and all of them agreed to be interviewed. The travel time from their home to the nearest health center ranged from 10 to 30 minutes. All were initially attended by a midwife. A summary of their profile is shown in Table 1.

Table 1. Profile of respondents $(n=14)$

\begin{tabular}{|c|c|}
\hline Age & $\begin{array}{l}\text { Range: } 24-43 \text { years old } \\
\text { Mean: } 32 \text { years old }\end{array}$ \\
\hline \multirow{3}{*}{ Gravidity } & Primigravida: $2(14 \%)$ \\
\hline & Multigravida: $12(86 \%)$ \\
\hline & Nullipara: $2(14 \%)$ \\
\hline \multirow[t]{3}{*}{ Parity } & Primipara: $4(29 \%)$ \\
\hline & Multipara: 8 (57\%) \\
\hline & Secondary level: $9(65 \%)$ \\
\hline \multirow[t]{2}{*}{ Highest educational attainment } & College level: $3(21 \%)$ \\
\hline & College graduate: $2(14 \%)$ \\
\hline \multirow{5}{*}{ Receiving facility } & Public tertiary care facility: $12(86 \%)$ \\
\hline & Private secondary care facility: $2(14 \%)$ \\
\hline & Hypertension: 4 (29\%) \\
\hline & Prolonged labor: $3(21 \%)$ \\
\hline & Big baby: $2(14 \%)$ \\
\hline \multirow{5}{*}{ Indication for referral } & Breech presentation: $1(7 \%)$ \\
\hline & Nausea and vomiting: $1(7 \%)$ \\
\hline & Threatened abortion: $1(7 \%)$ \\
\hline & Tuberculosis with malnutrition: $1(7 \%)$ \\
\hline & Intrauterine fetal death: $1(7 \%)$ \\
\hline \multirow[t]{4}{*}{ Outcome } & Vaginal delivery: $9(65 \%)$ \\
\hline & Caesarean section: $3(21 \%)$ \\
\hline & Partial breech extraction: $1(7 \%)$ \\
\hline & Intrauterine fetal death: $1(7 \%)$ \\
\hline
\end{tabular}

\subsection{Shared decision making}

Previous positive experience with the attending health professional is one of the consistently-mentioned factors that influence choice of health professional that will be consulted for the current pregnancy.

"I decided to continue with my current lying-in (clinic) because I had my previous delivery there.

I already know the midwife... There are nearer lying-in (clinics), but I already know the midwife in this center and she is kind."

Since mothers usually approach healthcare providers who are already familiar with them, they try to adhere with the advice given to them by having regular prenatal check-ups, taking medications and/or micronutrient supplements religiously, and attending buntis classes (i.e., information and education campaign for pregnant mothers). However, some barriers exist, such as familial obligations or lack of funds, which could prevent the mother from receiving optimum care. Another mother mentioned an incident where she was not consulted as to where she would like to be referred, and its consequences:

"I was monitored at the private lying in for four hours where I remained at $7 \mathrm{~cm}$. I was told that I needed to be brought to the hospital because my labor was not progressing. I was brought to a private hospital by the private midwife in their car. Their doctor requested for me to be admitted to their charity service and it is nearer than the public hospital. I waited for a bit before I was admitted but not too long. I was monitored for around 1 1/2 hours while I was being prepared for caesarean section. My cervix was stuck at 7-8 cm. The bag of water ruptured early while I was still at home and I am afraid I will have dry labor. I was not asked where I wanted to go. I was just brought there, but I was in the charity ward. I spent less than PhP 10,000 because we have PhilHealth. I did not expect to deliver via caesarean section. I was not prepared. We had to borrow money from different people to come up with the amount. Up to now, we are still in debt."

\subsection{Supportive environment}

The support of the partner or spouse, together with other members of the community, for the mother's pregnancy is seen to contribute to positive maternal outcomes. This is especially apparent in the formulation of a birth plan: 
"This is why I decided to deliver my youngest baby here. My midwife and I discussed my birth plan along with my husband. He usually accompanies me to my check-ups unless he has errands. He drives a tricycle."

Despite this, circumstances may prevent the implementation of a birth plan and the mother may have to rely on the support of other people:

"I have a birth plan but I was not able to follow it because my husband was out when I had labor. My mother-in-law accompanied me. My husband just followed."

Another anecdote shows that partners or spouses are not always supportive with the wishes of the mother:

"My husband initially consented to doing BTL (Bilateral Tubal Ligation), but changed his mind. We will just do condom and withdrawal because we do not have contact regularly. He usually defers to my wishes, but he is not sure that BTL is good for us."

\subsection{Service delivery}

The mothers have had a generally positive experience with the quality of care provided to them by the midwives who have seen them during their pregnancy. They consistently mentioned that the midwives remind them regularly regarding their birth plan and check-ups. One mother compared her latest experience in Legazpi with her previous pregnancy experience in Manila, the capital of the Philippines:

"The barangay health workers here are diligent in going around in reminding patients to have their check-ups and their vitamins and supplements. I did not experience that in Manila."

One mother narrated that there are times that continuity of care is not often achieved due to financial constraints:

"I had check-ups with this pregnancy; I think six. I had check-ups with a private doctor, but her professional fee for delivery was high so I decided to try doing it in a lying-in center. I had pre-natal check-ups at two different lying-in clinics."

For those who delivered at the public tertiary facility, satisfaction ranged from satisfied to highly dissatisfied:

"The doctors in the public tertiary hospital are good. But there are so many patients there. All patients from the whole of Bicol (Region) go there. I wish there are more hospitals here in Legazpi, but only the tertiary hospital has enough blood. There are private hospitals but most people here cannot afford to deliver there."

Many mothers reported negative experiences:

"I was accompanied by the midwife. I stayed in the tertiary facility for nine hours before I finally delivered. The doctors were rude to the midwife and me. They remarked that it was good that nothing wrong happened to your baby'. It is really difficult in the tertiary facility because there are so many patients.

Due to these experiences, some mothers remarked that it would have been better if they had just given birth at the local centers or lying-in clinics than in the tertiary hospital. This is especially highlighted by one mother, who compared her postpartum experience with her experiences during pregnancy and labor:

"We had the tagubilin once we were discharged, but I went back to the lying-in clinic for my postpartum and new born care. I prefer to go to the lying-in because the midwives are nice. And it is near my home."

The tagubilin is given to the mothers when they are discharged after giving birth. They are asked to give it to the facility that referred them for documentation and management during post-partum. However, mothers do not frequently return this. When probed why, they refused to give answers. One mother, who is privy to the workings of the local health system, remarked: 
"We have new government lying-in facilities. But more patients are going to the private facilities. They are given free food there. Sometimes, we are also given incentives as health workers to bring patients to the private lying-in. Even the tricycle drivers are paid around PhP 300-500 per patient. The government facilities are wasted because women opt to deliver in private lying-in clinics because of the perks."

\subsection{Discussion}

In general, mothers have expressed satisfaction with the patient-centeredness of the care they have received during their latest pregnancy. Healthcare decisions were often shared by the healthcare provider, the mother, and her immediate family. The mothers were supported by their spouses and other family members throughout the course of their pregnancy. Mothers were highly satisfied with the quality of care they have received during pregnancy and post-partum in primary care settings, but were mostly dissatisfied during labor in secondary and tertiary care facilities. Cost, patient disempowerment, negative attitudes of healthcare providers, inadequate staffing and health infrastructure were mentioned to prevent mothers from receiving optimum levels of patient-centered care. Perks afforded by private care facilities that may lead to undue influence in the decision to seek care were also noted.

Similar findings were encountered in other low- and middle-income settings. In a similar study conducted in Malawi, quality of care was satisfactory during antenatal care at primary care facilities, but declined during actual labor and delivery at tertiary facilities due to lack of hospital infrastructure [17]. Another study in Tanzania recounts overcrowding and lack of equipment and inadequate manpower, but also emphasizes the role of family in improving birth experience and bettering quality of care [18]. A separate study in Nigeria also found that while competent personnel and adequate equipment are present in public, tertiary hospitals, they are often inadequate and costly for the number of mothers giving birth at any point in time. However, some patients in the said study recounted that healthcare providers often take the extra effort to improve the non-medical aspects of care that is provided to them [19]. Lastly, problematic childbirth experiences did not seem to be present only in low- and middle-income countries. A study among UK first-time mothers recounted that they felt dehumanized on the first time they gave birth, indicating low quality of care [20].

A common theme from the results and from the literature to which the results are compared is that the quality of care being provided during labor and delivery is mostly poor. However, in an era where outcomes are measured in terms of patient satisfaction of the quality of care provided, it becomes imperative that a patient-centered perspective to providing care would be the mindset adapted by healthcare providers [11, 21]. This would lead to a more responsive, participative, and accessible healthcare system that could provide quality care in a timely and cost-effective manner. However, having this mindset would entail investments in health infrastructure and manpower to ensure that everybody receives an optimal level of care. In addition, these investments, should be coupled with improving how healthcare providers care for their patients [12, 22]. While healthcare providers often have a vision of what patient-centered care is, it may not be according to what is the standard definition [23]. This suggests that assessments of healthcare provider knowledge may be in order. Other strategies, such as regular audits, must be conducted to ensure that infrastructure, supplies, and manpower complement are always adequate, and protocols and guidelines are implemented [24]. At an organizational level, implementing agencies (i.e., Philippine Department of Health) should consider the adoption of the framework used in this study to assess its SDNs as it strives for the attainment of SDG targets and other program-and country-level objectives.

Having a systems approach to assessing quality of care allowed for a thorough review of adherence to referral protocols as stipulated in the guidelines. While most protocols were implemented well, there were several that these were not followed. The most apparent of these is the non-endorsement of the tagubilin to the referring facility. In the referral guidelines, it was emphasized that this should be returned by the mother to the referring center once she consults for postpartum and neonatal care. However, this is not strictly observed in practice. This may be due to the perception of both mothers and health workers that everything is fine once the mother has been discharged from the tertiary hospital. However, this would lead to incomplete records as this document might contain pertinent information regarding the patients' delivery and postpartum care. More importantly, instructions for postpartum and neonatal care written in the tagubilin may have been missed by the primary care facility, resulting to lower quality of care. These deficiencies show the necessity of training healthcare providers in adhering to protocols and guidelines to ensure better quality of care for both mother and new born child.

There may also be a need to investigate the practice of private facilities of giving dole-outs and perks to tricycle drivers and healthcare providers from public settings. On one side, such practices do not break any laws, provides extra income to these stakeholders, and prevents the mother from being hungry. On the other hand, they put undue influence on the patient to consult only private facilities, which would entail higher cost as compared to going to a public facility. It is also hypothesized that some private primary care facilities 
might have an agreement to send patients only to private hospitals. These areas of flexible legislation are perceived as opportunities in which undue influence and corruption can take root [25]. With these practices, patients are placed with a greater financial burden as compared to when they are referred to public hospitals, where services are cheaper or even free. In addition, this shows disempowerment in pursuing health-related choices, which results to dissatisfaction with quality of care.

In interpreting these conclusions, however, certain limitations should be noted. Due to our focus on the satisfaction of mothers, we were unable to corroborate our findings with other stakeholders. As such, we could only hypothesize regarding the misses of the healthcare provider in relation to their failure to collect tagubilin or whether or not private facilities had agreements with private hospitals in the area. Furthermore, the satisfactory remarks of patients regarding their antenatal care visits may be influenced by the fact that it was mostly conducted in the centre where they usually consult. They may be afraid to give unsatisfactory remarks in the presence of the people who attended to them, and other patients who may overhear their remarks during the interview. This has been identified as a possibility for explaining positive results in one previous study [26]. In subsequent studies, these may be remedied by interviewing them in more neutral locations so that mothers are not afraid to speak their mind.

Further research should build on the findings and the weaknesses of this study. A quantitative study may be done to describe how satisfied mothers are with the patient-centeredness of care they receive as they progress from pregnancy to postpartum, building on the findings of this study and other quantitative tools. Interviews should also be conducted with healthcare providers to triangulate what the mothers have said. These could then serve as major components, together with the aforementioned audits, of a monitoring and evaluation study to assess the implementation of this framework in SDNs. By adopting a patient-centered framework, the quality of care provided by SDNs to pregnant mothers will hopefully improve. This is seen to improve maternal outcomes at the patient and the population level, and hopefully contribute to attain SDG targets in a patient-centered manner at the time of universal healthcare.

\section{CONCLUSION}

The integrated patient-centered health service framework for maternal health can be used to assess the quality of care provided by SDNs. In this particular context, mothers were particularly satisfied with the quality of antenatal and postnatal care, but were less satisfied with the quality of care during labor and delivery, especially in tertiary centers. Factors that affected their overall experience include active participation in decision-making, supportive family and community, and provider attributes such as adherence to referral and management guidelines, respectful maternity care, physical comfort in the facility, and cost of care. Recommended strategies for improvement include training of healthcare providers regarding referral procedures and reorientation towards a patient-centered approach to care.

\section{ACKNOWLEDGEMENTS}

We would like to thank the mothers and healthcare providers that allowed this study to be conducted successfully. We would also like to express our sincerest gratitude to Cathy Banwell of the Australian National University, as well as to the editors and referees for their valuable inputs and comments to this manuscript.

\section{REFERENCES}

[1] Kassebaum N. J., Barber R. M., Bhutta Z. A., Dandona L., Gething P. W., Hay S. I., et al., "Global, regional, and national levels of maternal mortality, 1990-2015: a systematic analysis for the Global Burden of Disease Study 2015," The Lancet, vol. 388, no. 10053, pp. 1775-1812, 2016.

[2] Cagayan Ma S. F. S., Ricarte J. A., Ang-Bon R. M., Llamas-Clark E., "Insights on Maternal Health in the Philippines from National Health Surveys and Maternal Health Policies," Philippine Journal of Health Research and Development, vol. 22, no. 4, pp. 45-52, 2018.

[3] 15th Congress of the Philippines, "Responsible Parenthood and Reproductive Health Act," 2012. [Online]. Available: https://www.lawphil.net/statutes/repacts/ra2012/ra_10354_2012.html.

[4] Department of Health (Philippines), "Department of Health MNCHN Strategy Manual of Operations" 2011. [Online]. Available: https://www.doh.gov.ph/sites/default/files/publications/MNCHNMOPMay4withECJ.pdf.

[5] Department of Health (Philippines), "National Strategy towards Reducing Unmet Need for Modern Family Planning as a means to achieving MDGs on Maternal Health," 2012. [Online]. Available: https://ais.doh.gov.ph/uploads/aopdf/ao2012-0009.pdf.

[6] Department of Health (Philippines), "Provision of Blood Services under the Maternal, Neonatal, and Child Health and Nutrition (MNCHN) Strategy,” 2010. [Online]. Available: https://ais.doh.gov.ph/uploads/dcpdf/dc2010-0181.pdf. 
[7] Sangguniang Panlungsod (Council) of the City of Legazpi, "An ordinance establishing and adopting the commodity self-reliance (CSR) maternal newborn child health and nutrition (MNCHN) strategy for the effective and efficient implementation in the City of Legazpi," 0011-2014 2014 [Online]. Available: http://www.splegazpicity.com/ordinances_and_resolutions.

[8] World Health Organization, "Maternal mortality," 2018. [Online]. Available: https://www.who.int/news-room/factsheets/detail/maternal-mortality.

[9] Nieve, R, Jr. (2017). An analysis of maternal deaths in Camarines Norte: Points for action (PB 2017-03), [Online]. Available: https://zuelligfoundation.org/wp-content/uploads/2018/01/PB_Maternal-Death-Review-in-CamarinesNorte_v4.pdf

[10] Department of Health (Philippines), "Guidelines in Establishing Service Delivery Network,” [Online]. Available: https://www.doh.gov.ph/sites/default/files/publications/Guidelines\%20EstablishingSDN.pdf

[11] Kennedy H. P., Cheyney M., Dahlen H. G., Downe S., Foureur M. J., Homer C. S. E., et al., "Asking different questions: A call to action for research to improve the quality of care for every woman," Birth, vol. 45, no. 3, pp. 222-231, 2018.

[12] Sudhinaraset M., Afulani P., Diamond-Smith N., Bhattacharyya S., Donnay F., Montagu D., "Advancing a conceptual model to improve maternal health quality: The Person-Centered Care Framework for Reproductive Health Equity.," 2017. [Online]. Available: https://www.ncbi.nlm.nih.gov/pmc/articles/PMC5764229/.

[13] Gerteis M., Edgman-Levitan S., Daley J., Delbanco T. L., "Through the patient's eyes: Understanding and promoting patient-centered care," Jossey-Bass, 1993.

[14] Philippine Statistics Authority [Philippines], "2015 Census of Population-Region V,” 2015. [Online]. Available: https://www.psa.gov.ph/sites/default/files/attachments/hsd/pressrelease/R05.xlsx

[15] Cagayan, S., Ang-Bon, R., Salonga, R., Garcia, F.Jr., et.al., “ Analysis of trends in maternal mortalities in Bicol region using national health surveys and maternal death reviews," Philippine Journal of Obstetrics and Gynecology, vol.43, no.6, pp.39-48, 2019.

[16] NVivo 12. Melbourne, Australia: QSR International; 2018. [Online]. Available: https://www.qsrinternational.com/nvivo-qualitative-data-analysis-software/home

[17] Machira K., Palamuleni M., "Women's perspectives on quality of maternal health care services in Malawi," International Journal of Womens Health, vol. 10, pp. 25-34, 2018.

[18] Shimpuku Y., Patil C. L., Norr K. F., Hill P. D., "Women's Perceptions of Childbirth Experience at a Hospital in Rural Tanzania," Health Care for Women International, vol. 34, no. 6, pp. 461-481, 2013.

[19] Wright K., Banke-Thomas A., Sonoiki O., Ajayi B., Ilozumba O., Akinola O., "Opinion of women on emergency obstetric care provided in public facilities in Lagos, Nigeria: A qualitative study," Health Care for Women International, vol. 38, no. 6, pp. 527-534, 2017.

[20] Murphy H., Strong J., "Just another ordinary bad birth? A narrative analysis of first time mothers' traumatic birth experiences," Health Care for Women International, vol. 39, no. 6, pp. 619-634, 2018.

[21] Mead N., Bower P., "Patient-centredness: a conceptual framework and review of the empirical literature," Social Science and Medicine, vol. 51, no. 7, pp. 1087-1110, 2000.

[22] World Health Organization, "WHO|WHO Framework on integrated people-centred health services," 2019. [Online]. Available: http://www.who.int/servicedeliverysafety/areas/people-centred-care/en/.

[23] Adams T., Sarnak D., Lewis J., Convissar J., Young S. S., "What Do Clinicians Who Deliver Maternity Services Think Patient-Centered Care Is and How Is That Different for Vulnerable Women? A Qualitative Study,” 2018. [Online]. Available: https://www.hindawi.com/journals/jp/2018/5853235/.

[24] Saxena M., Srivastava A., Dwivedi P., Bhattacharyya S., "Is quality of care during childbirth consistent from admission to discharge? A qualitative study of delivery care in Uttar Pradesh, India," PLOS ONE, vol. 13, no. 9, 2018.

[25] Hernández-Aguado I., Chilet-Rosell E., "Pathways of undue influence in health policy-making: a main actor's perspective," Journal of Epidemiol and Community Health, vol. 72, no. 2, pp. 154-159, 2018.

[26] Grigoryan R., Thompson M. E., Crape B., Hekimian K., "Explaining Women's High Satisfaction with Objectively Poor Quality Childbirth Services: Armenia as a Case Study," Health Care for Women International, vol. 36, no. 1, pp. 121-134, 2015. 